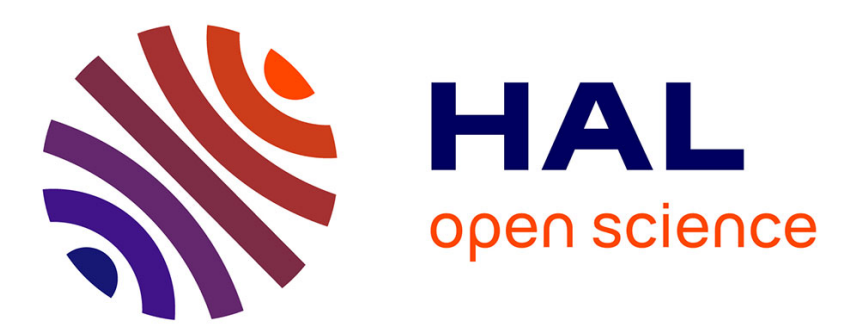

\title{
Processus aléatoire dans un réacteur nucléaire compte tenu des effets de la température du combustible I. Etablissement du modèle
}

\author{
R. Isnard
}

\section{- To cite this version:}

R. Isnard. Processus aléatoire dans un réacteur nucléaire compte tenu des effets de la température du combustible I. Etablissement du modèle. Revue de Physique Appliquée, 1973, 8 (1), pp.7-17. 10.1051/rphysap:01973008010700 . jpa-00243657

\section{HAL Id: jpa-00243657 https://hal.science/jpa-00243657}

Submitted on 1 Jan 1973

HAL is a multi-disciplinary open access archive for the deposit and dissemination of scientific research documents, whether they are published or not. The documents may come from teaching and research institutions in France or abroad, or from public or private research centers.
L'archive ouverte pluridisciplinaire HAL, est destinée au dépôt et à la diffusion de documents scientifiques de niveau recherche, publiés ou non, émanant des établissements d'enseignement et de recherche français ou étrangers, des laboratoires publics ou privés. 


\title{
PROCESSUS ALÉATOIRE DANS UN RÉACTEUR NUCLÉAIRE COMPTE TENU DES EFFETS DE LA TEMPÉRATURE DU COMBUSTIBLE ${ }^{*}$ ) I. ÉTABLISSEMENT DU MODẼLE
}

\author{
R. ISNARD $(* *)$ \\ Laboratoire de Génie Atomique de l'Ecole Polytechnique Fédérale de Lausanne
}

(Reçu le 23 novembre 1971, révisé le 25 juillet 1972)

\begin{abstract}
Résumé. - Une analyse probabiliste de la cinétique des réacteurs permet de décrire le comportement aléatoire des fluctuations, de certaines grandeurs physiques autour de leur valeur moyenne. Ces fluctuations peuvent être caractérisées, par exemple, par leur densité spectrale.

Dans ce travail, les populations des neutrons, des précurseurs et la température du combustible sont considérées comme des grandeurs aléatoires. Le processus décrivant le comportement du réacteur, qui est considéré comme ponctuel, est supposé markovien, ce qui permet de le décrire par l'équation de Chapman-Kolmogoroff. Les approximations de Fokker-Planck et de Langevin permettent de déterminer les densités spectrales énergétiques neutronique et thermique, ainsi que celle d'interaction neutrons-température du combustible.

Une analyse de ces dernières est effectuée afin de déterminer les domaines dans lesquels certains paramètres, comme la puissance du réacteur, le coefficient d'échange thermique et le coefficient de température du combustible, ont une influence.

Le temps de vie moyen des neutrons et le coefficient d'échange thermique peuvent se déduire à partir des densités spectrales.
\end{abstract}

\begin{abstract}
A stochastic analysis of reactor Kinetics allows a description of random fluctuations of some physical variables around their mean values. These fluctuations can be characterized, for instance, by their spectral density.

In this paper, the number of neutrons, of precursor nucleis and the fuel temperature are random variables. The stochastic process occuring in a nuclear reactor can be described by a space and energy independent markovian random process. The Chapman-Kolmogoroff equation, then the Fokker-Planck and the Langevin approximations allow one to calculate the spectral densities of the fluctuations of the number of neutrons, of the fuel temperature and of the cross power spectral density of these two kinds of fluctuations.

An analysis of these densities is made in order to know the field in which some parameters, such as reactor-power, heat transfert coefficient and fuel-temperature coefficient, have an effect.

The mean lifetime of neutrons and the heat transfert coefficient can be, for instance, estimated from spectral densities.
\end{abstract}

1. Introduction. - Le comportement d'un réacteur peut être analysé de deux manières différentes :

- soit en le considérant comme décrit par jun processus aléatoire,

- soit en le considérant comme décrit par un processus déterministe.

Dans la présente étude, nous avons choisi le premier type d'analyse.

On supposera que le réacteur est de faible puissance $(<100 \mathrm{~kW})$ afin de pouvoir fonctionner avec un régime de refroidissement par circulation naturelle,

(*) Extrait du travail présenté à l'Ecole Polytechnique Fédérale de Lausanne pour l'obtention du grade de docteur ès sciences techniques.

(**) Actuellement au CEA/DAM du Centre d'Etudes de Valduc (SECR). ou forcée, mais de faible débit, de manière à limiter les vibrations mécaniques; en effet, ces dernières introduisent des perturbations gênantes qui ne peuvent être prises en considération dans l'établissement du modèle.

Le développement théorique est celui introduit par A. Dalfes [1], [2], pour la détermination de l'équation de Fokker-Planck, des équations différentielles des fonctions de corrélation et des covariances. A partir de ces dernières, cet auteur détermine les densités spectrales par une méthode numérique [2], tandis que dans le présent travail nous expliciterons ces densités spectrales par une application directe de la méthode de M. Lax [3]. Le modèle décrit par A. Dalfes ne comporte pas de contre-réaction, nous en introduirons une ici, due à l'influence de la température du combustible. 
Nous admettrons que les propriétés nucléaires du réacteur dépendent de la température du combustible par l'intermédiaire des probabilités de fission et de disparition (capture stérile, fuite hors du réacteur) comme nous le verrons dans le chapitre 2 .

Dans leurs articles, D. R. Harris [4] et P. R. Pluta [5] ont également introduit une contre-réaction due à l'effet de la température du combustible, mais uniquement sur la probabilité de disparition ; d'autre part, ils ne se sont intéressés qu'à l'étude des moments du premier et du second ordre, par la méthode de la fonction génératrice de probabilité, en vue uniquement de la détermination de critères de stabilité du réacteur. Dans le chapitre 3 on trouvera, en plus de ces moments, les densités spectrales énergétiques relatives à la population des neutrons, à la température du combustible et la densité spectrale d'interaction neutrons-température, qui serviront à l'évaluation de paramètres caractéristiques du réacteur.

K. Saito [6], [7] décrit le comportement du réacteur par la relation d'Einstein, généralisée par M. Lax, en tenant compte des contre-réactions dues aux effets de températures du combustible et du réfrigérant, qui comprend le modérateur, sur les sections efficaces. Cet auteur s'intéresse aux sources de bruit génératrices de fluctuations du niveau de puissance.

Pour établir le modèle étudié dans cet article, nous avons choisi un ensemble de variables aléatoires tel qu'une description markovienne du processus caractérisant le comportement du réacteur soit possible.

2. Bilan des probabilités. Hypothèses de linéarisation. Equations des moments. - L'évolution temporelle du réacteur, supposé ponctuel, peut être décrite par l'équation de Chapman-Kolmogoroff [8] :

$$
\begin{aligned}
P(\mathbf{y}, t+\delta t & \left.\mid \mathbf{y}_{0}, t_{0}\right)= \\
= & \sum_{\mathbf{y}^{\prime}=0}^{M} P\left(\mathbf{y}^{\prime}, t \mid \mathbf{y}_{0}, t_{0}\right) \cdot P\left(\mathbf{y}^{\prime} \rightarrow \mathbf{y}, \delta t\right)
\end{aligned}
$$

dans laquelle :

$$
P\left(\mathbf{y}, t+\delta t \mid \mathbf{y}_{0}, t_{0}\right)
$$

probabilité conditionnelle de l'état $\mathbf{y}$ au temps $t+\delta t$, étant donné l'état $\mathbf{y}_{0}$ au temps $t_{0}$;

$$
P\left(\mathbf{y}^{\prime}, t \mid \mathbf{y}_{0}, t_{0}\right):
$$

probabilité conditionnelle de l'état $\mathbf{y}^{\prime}$ au temps $t$, étant donné l'état $\mathbf{y}_{0}$, au temps $t_{0}$;

$$
P\left(\mathbf{y}^{\prime} \rightarrow \mathbf{y}, \delta t\right)=P\left(\mathbf{y}, t+\delta t \mid \mathbf{y}^{\prime}, t\right):
$$

probabilité de transition de l'état $\mathbf{y}^{\prime}$ vers $\mathbf{y}$ pendant $\delta t$.

A l'équation précédente est liée la condition initiale suivante :

$$
\lim _{t \rightarrow t_{0}} P\left(\mathbf{y}, t \mid \mathbf{y}_{0}, t_{0}\right)=\delta\left(\mathbf{y}, \mathbf{y}_{0}\right)
$$

$\operatorname{avec} \delta\left(\mathbf{y}, \mathbf{y}_{0}\right)$ symbole de Kronecker.
Le vecteur des variables aléatoires $\mathbf{y}$ représentant l'état du réacteur a les composantes suivantes :

$\mathbf{y}^{T}=\left(N, C, \theta_{\mathrm{u}}\right)$

$N=$ population totale des neutrons (théorie à un groupe d'énergie);

$C=$ population totale des précurseurs, émetteurs de neutrons retardés (théorie à un groupe de neutrons retardés);

$\theta_{\mathrm{u}}=T_{\mathrm{u}} \cdot C_{\mathrm{u}} / q$ si $T_{\mathrm{u}}$ est la température moyenne spatiale du combustible, $C_{\mathrm{u}}$ la chaleur spécifique massique totale du combustible et $q$ la quantité moyenne d'énergie apportée par fission.

L'énergie thermique est ainsi quantifiée et $\theta_{\mathrm{u}}$ représente le nombre total d'unités d'énergie interne contenues dans le combustible.

Le réfrigérant est considéré comme un réservoir de chaleur infini à une température moyenne spatiale connue $T_{\mathrm{m}_{0}}$. Cette hypothèse est d'autant plus justifiée que la masse du réfrigérant est importante (ce qui est notamment le cas des réacteurs du type piscine) et que le système de refroidissement est efficace. Nous pouvons également définir le nombre total d'unités d'énergie interne contenues dans le réfrigérant: $\theta_{\mathrm{m}_{\mathrm{o}}}=T_{\mathrm{m}_{0}} \cdot C_{\mathrm{u}} / q$.

Ayant défini les composantes du vecteur $\mathbf{y}$, nous allons expliciter toutes les transitions possibles pour les différentes variables aléatoires, entre les instants $t$ et $t+\delta t$, et les probabilités de transitions correspondantes. Pour ce faire, nous adoptons l'hypothèse suivante :

2.1. HypothèSE $1 .-\delta t$ est choisi tel que la probabilité d'observer deux événements pendant $\delta t$ est négligeable devant la probabilité d'en observer un seul.

Nous pouvons alors définir les probabilités de transitions suivantes:

1. Capture non productive de fission et fuite hors du réacteur: soit $\alpha_{1}(t)$ la probabilité par unité de temps et par neutron pour que ce dernier subisse la capture stérile, ou fuie hors du réacteur (taux de disparition des neutrons)

2. Décroissance des précurseurs : soit $\lambda$ la probabilité par unité de temps et par précurseur pour que ce dernier décroisse et donne un neutron retardé (taux d'apparition des neutrons retardés et de disparition des précurseurs).

3. Fission : soit $\alpha_{f}(t)$ la probabilité par unité de temps et par neutron pour que ce dernier crée la fission (taux de production des neutrons prompts et des précurseurs). Soit également $P(\boldsymbol{v})=P\left(v_{0}, v_{\mathrm{i}}\right)$ la probabilité indépendante du temps et de l'énergie des neutrons, pour que $v_{0}$ neutrons, $v_{\mathbf{i}}$ précurseurs soient créés à la fission. $v_{0}, v_{\mathrm{i}}$ sont des valeurs entières et non négatives.

$$
\sum_{v=0}^{\infty} P(v)=1
$$


4. Emission de neutrons par une source extérieure (ou de fission spontanée) : soit $S(t)$ la probabilité par unité de temps pour que la source émette un neutron (taux d'immigration des neutrons).

5. Perte d'unités d'énergie interne du combustible: soit $\alpha_{\theta}(t)$ la probabilité par unité de temps et par unité d'énergie interne du combustible pour que le nombre de ces dernières décroisse d'une unité (taux de transmission d'énergie du combustible au réfrigérant).

6. Gain d'unités d'énergie interne du combustible : soit $\alpha_{\mathrm{m}}(t)$ la probabilité par unité de temps et par unité d'énergie interne du réfrigérant pour que celles du combustible s'accroissent d'une unité (taux de transmission d'énergie du réfrigérant au combustible).

7. Aucune des transitions décrites de 1 à 6 n'a lieu pendant $\delta t$.

Le schéma ci-contre illustre les sept transitions considérées.

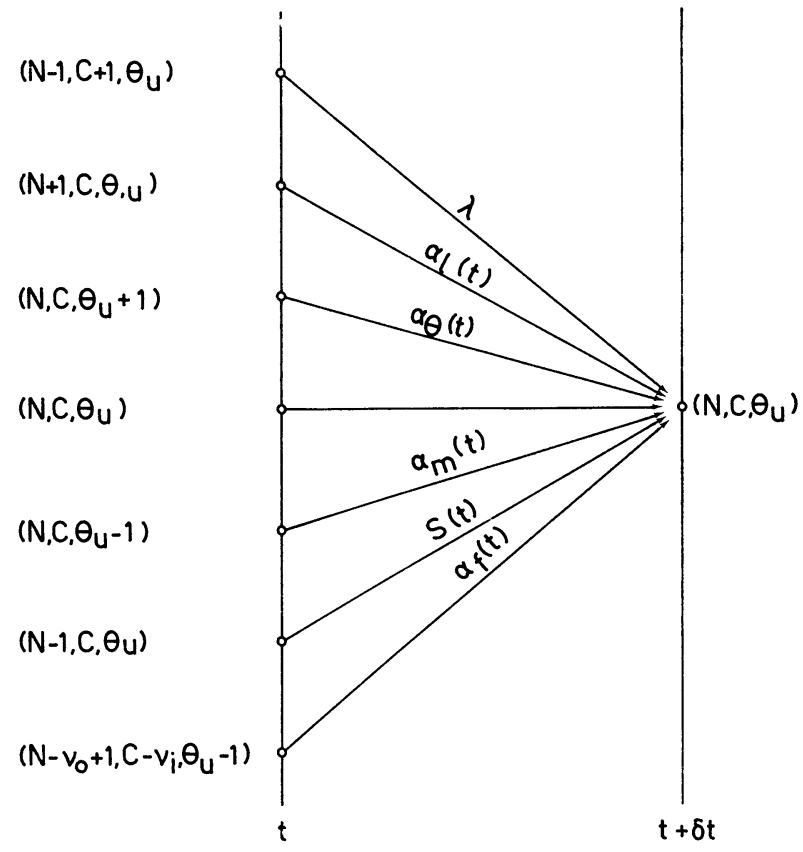

FIG. 1.

L'équation (1) nous permet alors d'écrire en considérant les sept transitions ci-dessus citées le bilan des probabilités suivant: [1], [4]

$$
\begin{aligned}
P(N, & \left.C, \theta_{\mathrm{u}}, t+\delta t \mid N_{0}, C, \theta_{u_{0}}, t_{0}\right)=\alpha_{1}(t) \cdot(N+1) \cdot P\left(N+1, C, \theta_{\mathrm{u}}, t \mid N_{0}, C_{0}, \theta_{\mathrm{u}_{0}}, t_{0}\right) \cdot \delta t+ \\
& +\lambda \cdot(C+1) \cdot P\left(N-1, C+1, \theta_{\mathrm{u}}, t \mid N_{0}, C_{0}, \theta_{\mathrm{u}_{0}}, t_{0}\right) \cdot \delta t \\
& +\sum_{\mathbf{v}=0}^{\infty} P(v) \cdot \alpha_{\mathrm{f}}(t) \cdot\left(N-v_{0}+1\right) \cdot P\left(N-v_{0}+1, C-v_{i}, \theta_{\mathrm{u}}-1, t \mid N_{0}, C_{0}, \theta_{\mathrm{u}_{0}}, t_{0}\right) \\
& +S(t) \cdot P\left(N-1, C, \theta_{\mathrm{u}}, t \mid N_{0}, C_{0}, \theta_{\mathrm{u}_{0}}, t_{0}\right) \cdot \delta t \\
& +\alpha_{\theta}(t) \cdot\left(\theta_{\mathrm{u}}+1\right) \cdot P\left(N, C, \theta_{\mathrm{u}}+1, t \mid N_{0}, C_{0}, \theta_{\mathrm{u}_{0}}, t_{0}\right) \cdot \delta t \\
& +\alpha_{\mathrm{m}}(t) \cdot \theta_{\mathrm{m}_{0}} \cdot P\left(N, C, \theta_{\mathrm{u}}-1, t \mid N_{0}, C_{0}, \theta_{\mathrm{u}_{0}}, t_{0}\right) \cdot \delta t \\
& +\left\{1-\left[S(t)+\lambda \cdot C+\left[\alpha_{1}(t)+\alpha_{\mathrm{f}}(t)\right] \cdot N+\alpha_{\theta}(t) \cdot \theta_{\mathrm{u}}+\alpha_{\mathrm{m}}(t) \cdot \theta_{\mathrm{m}_{0}}\right] \cdot \delta t\right\} P\left(N, C, \theta_{\mathrm{u}}, t \mid N_{0}, C_{0}, \theta_{\mathrm{u}_{0}}, t_{0}\right) .
\end{aligned}
$$

En faisant passer $P\left(N, C, \theta_{\mathrm{u}}, t \mid N_{0}, C_{0}, \theta_{\mathrm{u}_{0}}, t_{0}\right)$ dans le membre de gauche de (4), en divisant les deux membres par $\delta t$ et en faisant tendre $\delta t$ vers zéro, on obtient l'équation différentielle du bilan des probabilités, qui est l'équation de Chapman-Kolmogoroff.

Pour résoudre cette équation, nous faisons l'hypothèse suivante :

2.2. НуротнѐSE 2. - Dans un réacteur, la population des neutrons, celle des précurseurs, et le nombre d'unités d'énergie interne contenues dans le combustible sont grands par rapport à leurs variations dues à la fission, la capture, les fuites et les immigrations. On peut donc considérer ces variations comme des changements infinitésimaux des populations des neutrons, des précurseurs et du nombre d'unités d'énergie interne contenues dans le combustible. En conséquence, le processus de Markoff décrivant le comportement du réacteur peut être considéré comme continu dans les espaces du temps et du vecteur d'état $\mathbf{y}$.
Cette hypothèse conduit à l'équation de FokkerPlanck et de là aux systèmes d'équations différentielles des moments du premier ordre, des covariances et des fonctions de corrélation. Afin d'alléger la présentation de ce travail, nous avons reporté dans l'annexe A le détail de ces calculs.

Ces systèmes d'équations peuvent être résolus compte tenu des trois hypothèses de linéarisation suivantes :

2.3. НyPOTHÈSE 3. - Les propriétés nucléaires du réacteur varient linéairement avec la température du combustible. C'est-à-dire que l'on peut exprimer les probabilités de transition $\alpha_{\mathrm{f}}(t)$ et $\alpha_{1}(t)$, de la manière suivante :

$$
\begin{aligned}
& \alpha_{1}(t)=\alpha_{1_{0}}(t)-\gamma_{1} \cdot\left[\theta_{\mathrm{u}}-\theta_{\mathrm{u}_{0}}\right] \\
& \alpha_{\mathrm{f}}(t)=\alpha_{\mathrm{f}_{0}}(t)-\gamma_{2} \cdot\left[\theta_{\mathrm{u}}-\theta_{\mathrm{u}_{0}}\right] .
\end{aligned}
$$

Les propriétés nucléaires du réacteur sont supposées connues pour la température $T_{\mathrm{u}_{0}}$. Les coefficients $\gamma_{1}$ et $\gamma_{2}$ sont des coefficients de température agissant 
respectivement sur la disparition des neutrons (effet de densité, de fuite et effet Doppler) et sur la fission (effet de densité et effet Doppler).

Cette dépendance de la température des probabilités de transitions $\alpha_{\mathrm{f}}(t)$ et $\alpha_{1}(t)$ entraîne une nonlinéarité des systèmes d'équations différentielles cidessus cités.

La linéarisation est obtenue à l'aide des deux hypothèses suivantes :

2.4. Нypothèse 4. - Les densités de probabilité conditionnelle $p\left(\mathbf{y}, t \mid \mathbf{y}_{0}, t_{0}\right)$ et les densités de "probabilité jointe » $p\left(\mathbf{y}^{2}, t_{2} ; \mathbf{y}^{1}, t_{1}\right)$ tendent vers une loi normale que l'on peut justifier du fait que les variables d'état y sont grandes.

En effet, les nombres de neutrons, de précurseurs et d'unités d'énergie interne contenues dans le combustible sont grands pour un réacteur fonctionnant même à très faible puissance.

Cette hypothèse permet d'exprimer les moments du troisième ordre, apparaissant dans les équations différentielles des covariances et des fonctions de corrélation, en fonction de ceux du deuxième et $\mathrm{du}$ premier ordre. L'apparition de ces moments du troisième ordre est due à la non-linéarité introduite par l'hypothèse 3 . Nous sommes ainsi ramenés à la réso- lution de systèmes d'équations dans lesquels le nombre d'inconnues est égal au nombre d'équations.

2.5. НуротнѐSE 5. - Le processus markovien décrit par le vecteur des variables aléatoires y est stationnaire.

Cela implique que les probabilités de transition sont indépendantes du temps, les «probabilités jointes» de plusieurs variables ne dépendent que des intervalles de temps $t_{2}-t_{1}=\tau$, les probabilités conditionnelles sont indépendantes du temps. Les fonctions de corrélation ne dépendent plus que de $\tau$, et non plus des instants particuliers considérés, et les dérivées par rapport au temps des moments du premier ordre et des covariances sont nulles.

Nous pouvons alors écrire:

1. Equations des fonctions de corrélation:

$$
\frac{\mathrm{d}}{\mathrm{d} \tau} \Phi(\tau)=\Phi(\tau) \cdot \Lambda^{T} \quad(\tau>0)
$$

avec :

$$
\Phi(\tau)=\left[\varphi_{k l}(\tau)\right] k, l=N, C, \theta_{\mathrm{u}}
$$

matrice des fonctions de corrélation (voir annexe $\mathrm{A}$ ), $\Lambda^{\mathrm{T}}$ : matrice transposée de la matrice $\Lambda$ définie cidessous,

$\Lambda$ : est appelée «matrice du vecteur vitesse ».

$$
\begin{aligned}
\Lambda=\left[\begin{array}{r}
{\left[\alpha_{\mathrm{f}_{0}} \cdot\left(<v_{0}>-1\right)-\alpha_{\mathrm{l}_{0}}-\gamma_{\mathrm{u}} \cdot\left(<\theta_{\mathrm{u}}>\right.\right.} \\
\beta \cdot<v>\cdot\left[\alpha_{\mathrm{f}_{0}}-\gamma_{2} \cdot\left(<\theta_{\mathrm{u}}>\right.\right. \\
{\left[\alpha_{\mathrm{f}_{0}}-\gamma_{2} \cdot\left(<\theta_{\mathrm{u}}>\right.\right.}
\end{array}\right. \\
<v>=<v_{0}>+\beta<v>, \\
\left\langle v_{\mathrm{i}}\right\rangle=\beta<v>, \\
\langle v>=\text { nombre moyen total de neutrons émis par } \\
\quad \text { fission, } \\
\beta \quad=\text { fraction totale des neutrons retardés, } \\
\gamma_{\mathrm{u}} \quad=\left(<v_{0}>-1\right) \cdot \gamma_{2}-\gamma_{1} .
\end{aligned}
$$

L'équation (6) doit être résolue avec la condition aux limites :

$$
\lim _{\tau=0} \Phi(\tau)=M .
$$

La matrice $M$ est déterminée par l'équation des covariances suivante:

\section{Equations des covariances:}

$$
\frac{\mathrm{d}}{\mathrm{d} t} M(t)=M(t) \cdot \Lambda^{T}+\Lambda \cdot M(t)+<\Sigma>=0
$$

avec :

$$
M(t)=\left[\mu_{k l}(t)\right] k, l=N, C, \theta_{\mathbf{u}},
$$

matrice des covariances au temps $t$;

$$
M(t)=M
$$

d'après l'hypothèse 5 (voir annexe $\mathrm{A}$ );

$$
\left.\langle\Sigma\rangle=\left[\sigma_{k l}(<\mathbf{y}\rangle\right)\right] k, l=N, C, \theta_{\mathrm{u}},
$$

«matrice de diffusion" (voir annexe $\mathrm{A}$ ).

Remarques. - $a$. L'éq. matricielle (10) est identique à celle trouvée par D. R. Harris [4], compte tenu de l'hypothèse 4.

$b$. L'éq. (6) avec la condition (9) peut être résolue numériquement par la méthode des valeurs propres, comme le préconise A. Dalfes [2].

3. Equations des moments du premier ordre (Valeurs moyennes). - Le comportement moyen des variables d'état $N, C, \theta_{\mathrm{u}}$ peut se mettre sous la forme suivante :

$$
\begin{gathered}
0=\left[\alpha_{\mathrm{f}_{0}} \cdot\left(<v_{0}>-1\right)-\alpha_{\mathrm{l}_{0}}-\gamma_{\mathrm{u}} \cdot\left(<\theta_{\mathrm{u}}>-\theta_{\mathrm{u}_{0}}\right)\right] \times \\
\times<N>+\lambda \cdot<C>+<S>-\gamma_{\mathrm{u}} \cdot \mu_{N \theta_{\mathrm{u}}} \\
0=\beta \cdot<v>\cdot\left[\alpha_{\mathrm{f}_{0}}-\gamma_{2} \cdot\left(<\theta_{\mathrm{u}}>-\theta_{\mathrm{u}_{0}}\right)\right] \times \\
\times<N>-\lambda \cdot<C>-\beta \cdot<v>\cdot \gamma_{2} \cdot \mu_{N \theta_{\mathrm{u}}} \\
0=\left[\alpha_{\mathrm{f}_{0}}-\gamma_{2} \cdot\left(<\theta_{\mathrm{u}}>-\theta_{\mathrm{u}_{0}}\right)\right] \cdot<N>-\alpha_{\theta} \times \\
\times<\theta_{\mathrm{u}}>+\alpha_{\mathrm{m}} \cdot \theta_{\mathrm{m}_{0}}-\gamma_{2} \cdot \mu_{N \theta_{\mathrm{u}}} .
\end{gathered}
$$


Nous remarquons que dans les équations des moments du premier ordre (valeurs moyennes) des variables d'état, apparaissent les moments du deuxième ordre : $\mu_{N \theta_{u}}$. Ceci est dû au fait que les probabilités de transition $\alpha_{\mathrm{f}}$ et $\alpha_{1}$ dépendent de la température.

Ces équations sont identiques à celles trouvées par D. R. Harris [4], K. Saito [6] et P. R. Pluta [5].

Si nous faisons $\gamma_{1}=\gamma_{2}=0$, nous retrouvons les équations classiques de la cinétique des réacteurs en théorie ponctuelle à un groupe d'énergie et à un groupe de neutrons retardés, ainsi que les équations du bilan d'énergie entre le combustible et le réfrigérant en régime permanent.
Au chapitre 3, nous allons exprimer, par la méthode de Langevin [3] exposée dans l'annexe B, les densités spectrales énergétiques des fluctuations de la population des neutrons $s_{N N}(f)$, des fluctuations de la température du combustible $s_{\theta_{\mathrm{u}} \theta_{\mathrm{u}}}(f)$ et la densité spectrale d'interaction neutrons-température $s_{N \theta_{\mathrm{u}}}(f)$.

3. Densités spectrales énergétiques et d'interaction. - 3.1. DENSITÉ SPECTRALE ÉNERGÉTIQUE NEUTRONIQUE. - Le premier élément diagonal de la matrice $S(f)$ définie dans l'annexe $\mathbf{B}$, donne la densité spectrale énergétique des fluctuations de la population des neutrons. Nous avons remplacé $2 \pi f$ par $\omega$ pour simplifier l'écriture :

$$
\begin{aligned}
s_{N N}(f)=\frac{\langle N\rangle}{a^{2}+\omega^{2} \cdot b^{2}} & \cdot\left\{[ \omega ^ { 2 } + ( \alpha _ { \theta } + \gamma _ { 2 } \cdot < N > ) ^ { 2 } ] \cdot \left[\left(\omega^{2}+\lambda^{2}\right) \cdot \alpha_{t}+\right.\right. \\
+ & \left.\lambda^{2} \cdot \alpha_{\mathrm{f}} \cdot\left[\beta \cdot<v \cdot(\beta \cdot<v>+1)>+2 \beta \cdot<v \cdot\left(v_{0}-2\right)>\right]\right] \\
& -2 \beta \cdot<v>\cdot \alpha_{\mathrm{f}} \cdot \lambda \cdot \gamma_{\mathrm{u}} \cdot\left\langle N>\cdot\left(\omega^{2}+\alpha_{\theta} \cdot \lambda\right)\right. \\
& -2\left(<v_{0}>-1\right) \cdot \alpha_{\mathrm{f}} \cdot \gamma_{\mathrm{u}} \cdot<N>\cdot\left(\alpha_{\theta}+\gamma_{2} \cdot<N>\right) \cdot\left(\omega^{2}+\lambda^{2}\right) \\
& -2(\beta \cdot<v>)^{2} \cdot \alpha_{\mathrm{f}} \cdot \lambda^{2} \cdot\left(\alpha_{\theta}+\gamma_{2} \cdot<N>\right) \cdot \gamma_{2} \cdot<N> \\
+ & 2 \beta \cdot<v>\cdot\left(<v_{0}>-1\right) \cdot \alpha_{\mathrm{f}} \cdot \lambda \cdot \gamma_{2} \cdot<N>\cdot\left[\omega^{2}-\lambda \cdot\left(\alpha_{\theta}+\gamma_{2} \cdot<N>\right)\right] \\
& +2\left(\alpha_{\mathrm{f}} \cdot<N>+\alpha_{\mathrm{m}} \cdot \theta_{\mathrm{m}_{0}}\right) \cdot\left[\beta \cdot<v>\cdot \lambda^{2} \cdot \gamma_{\mathrm{u}} \cdot \gamma_{2} \cdot<N>+\left(\omega^{2}+\lambda^{2}\right) \cdot \gamma_{\mathrm{u}}^{2} \cdot<N>\right. \\
& \left.\left.+(\beta \cdot<v>)^{2} \cdot \lambda^{2} \cdot \gamma_{2}^{2} \cdot<N>\right]\right\}
\end{aligned}
$$

avec :

$$
\begin{aligned}
a & =-\left\{\omega ^ { 2 } \cdot \left(\alpha_{\mathrm{p}}+\lambda+\alpha_{\theta}+\gamma_{2} \cdot\langle N>)-\lambda \cdot\left[\alpha _ { \mathrm { p } } \cdot \left(\alpha_{\theta}+\gamma_{2} \cdot\langle N>)+\alpha_{\mathrm{f}} \cdot \gamma_{\mathrm{u}} \cdot\left\langle N>-\beta \cdot\left\langle v>\cdot \alpha_{\mathrm{f}} \cdot \alpha_{\theta}\right]\right\}\right.\right.\right.\right. \\
b & =\omega^{2}-\alpha_{\mathrm{p}} \cdot\left(\lambda+\alpha_{\theta}+\gamma_{2} \cdot\langle N>)+\alpha_{\mathrm{f}} \cdot\left(\beta \cdot \left\langlev>\cdot \lambda-\gamma_{\mathrm{u}} \cdot\langle N>)-\lambda \cdot\left(\alpha_{\theta}+\gamma_{2} \cdot\langle N>)\right.\right.\right.\right. \\
\alpha_{\mathrm{p}} & =-\left[\left(\left\langle v_{0}>-1\right) \cdot \alpha_{\mathrm{f}}-\alpha_{1}\right]\right. \\
\alpha_{\mathrm{t}} & =\left\langle v_{0} \cdot\left(v_{0}-1\right)>\cdot \alpha_{\mathrm{f}}+2 \alpha_{\mathrm{p}}\right.
\end{aligned}
$$

$s_{N N}(f)$ n'est plus seulement proportionnel à $\langle N\rangle$ (cas sans contre-réaction de température), mais également à $\langle N\rangle^{2}$ et $\langle N\rangle^{3}$; toutefois l'influence de ces deux derniers termes ne se fait sentir que pour les très basses fréquences $\left(f<10^{-2} \mathrm{~Hz}\right)$ et pour des puissances du réacteur de l'ordre de plusieurs kilowatts [10]. Pour ces puissances de fonctionnement une augmentation du coefficient de température du combustible qui est supposé négatif peut entraîner une diminution importante de l'amplitude des fluctuations de la population totale des neutrons surtout si le coefficient d'échange thermique $\gamma$ est faible (cas de la convection naturelle).

Sur la figure 2 nous avons représenté d'une part, en trait plein, la densité spectrale énergétique neutronique sans contre-réaction de température et d'autre part, celles avec contre-réaction pour des coefficients de température du combustible compris entre $-0,1$ et $-100 \mathrm{pcm} /{ }^{\circ} \mathrm{K}$. Le réacteur est supposé fonctionner à environ $50 \mathrm{~kW}$, refroidi en régime de convection naturelle.

Jusqu'à présent les moyennes intervenant dans toutes les expressions étaient des moyennes d'ensem-

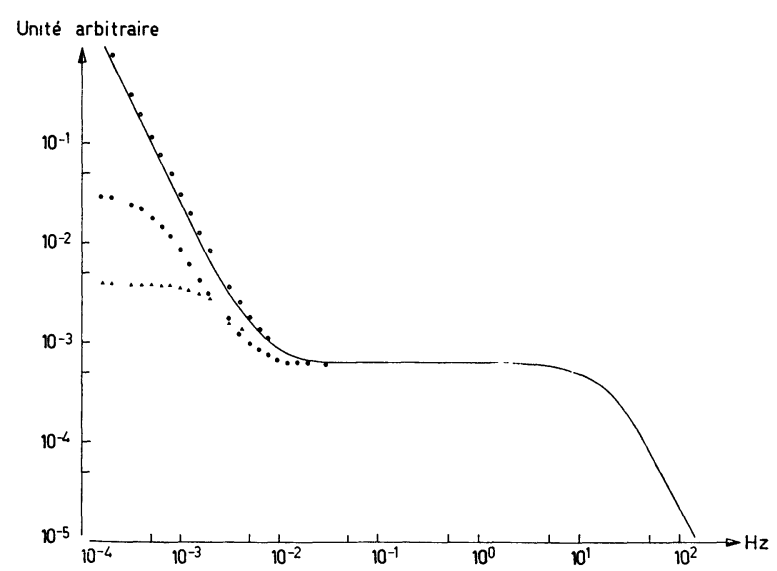

FIG. 2.

$\langle N\rangle=2,5 \times 10^{11}\left(P_{\text {react }} \cong 50 \mathrm{~kW}\right)$

$l=65 \mu \mathrm{s}$

$\beta=800 \mathrm{pcm}$

$\lambda=0,05 \mathrm{rd} / \mathrm{s}$

$\gamma=200 \mathrm{~W} / \mathrm{m}^{2} \quad \mathrm{~K}$

Cas sans contre-réaction

$\bigcirc \quad \partial \rho / \partial T_{\mathrm{u}}=-0,1 \mathrm{pcm} /{ }^{\circ} \mathrm{K}$

- $\partial \rho / \partial T_{\mathrm{u}}=-10 \mathrm{pcm} /{ }^{\circ} \mathrm{K}$

$\partial \rho / \partial T_{\mathrm{u}}=-100 \mathrm{pcm} /{ }^{\circ} \mathrm{K}$ 
bles. En supposant que l'hypothèse d'ergodicité soit applicable, l'expression (12) peut se simplifier en considérant des fréquences supérieures à 1 hertz.

En effet, nous pouvons montrer facilement que : [1], [4], [5], [6], [7]

$$
\begin{aligned}
\text { - } \rho & =\frac{\alpha_{\mathrm{f}} \cdot(\bar{v}-1)-\alpha_{1}}{\bar{v} \cdot \alpha_{\mathrm{f}}} \text { est la réactivité } \\
\text { - } \quad \alpha_{\mathrm{p}} & =-(\rho-\beta) \cdot \frac{k_{\mathrm{e}}}{l}=-\frac{(\rho-\beta)}{\operatorname{tg}}
\end{aligned}
$$

avec :

$\beta$ fraction totale des neutrons retardés,

$\bar{v}$ nombre total moyen de neutrons émis par fission,

$k_{\mathrm{e}}$ facteur de multiplication effectif,

$l$ temps de vie moyen des neutrons,

tg temps de génération des neutrons

$$
\begin{aligned}
\alpha_{\mathrm{f}} & =\frac{k_{\mathrm{e}}}{\bar{v} \cdot l} \\
\alpha_{\theta} & =\gamma \cdot \frac{S_{\mathrm{e}}}{C_{\mathrm{u}}}
\end{aligned}
$$

avec :

$\gamma$ coefficient d'échange thermique,

$S_{\mathrm{e}} \quad$ surface totale d'échange entre le combustible et le réfrigérant,

$C_{\mathrm{u}}$ chaleur spécifique massique totale du combustibıe,

$$
\begin{gathered}
\alpha_{\mathrm{m}}=\alpha_{\theta} \\
-\quad \frac{\delta \rho}{\delta T_{\mathrm{u}}} \simeq-\frac{C_{\mathrm{u}}}{q} \cdot \frac{\gamma_{\mathrm{u}} \cdot l}{k_{\mathrm{e}}}
\end{gathered}
$$

est le coefficient de température du combustible.

Compte tenu des expressions (17) à (22), (12) peut s'écrire pour $f>1$ :

$$
s_{N N}(f)=\frac{\left[\omega^{2}+\left(\alpha_{\theta}+\gamma_{2} \cdot \bar{N}\right)\right] \cdot\left[\omega^{2}+\lambda^{2}\right] \cdot \alpha_{\mathrm{t}} \cdot \bar{N}}{\left[-\omega^{2} \cdot\left(\alpha_{\mathrm{P}}+\alpha_{\theta}+\gamma_{2} \cdot \bar{N}+\lambda\right)\right]^{2}+\omega^{2} \cdot\left[\omega^{2}-\alpha_{\mathrm{P}} \cdot\left(\alpha_{\theta}+\gamma_{2} \cdot \bar{N}+\lambda\right)\right]^{2}} .
$$

Et comme :

$$
\begin{aligned}
& \alpha_{\mathrm{p}} \gg\left(\alpha_{\theta}+\gamma_{2} \cdot \bar{N}\right) \quad, \quad \alpha_{\mathrm{p}} \gg \lambda \\
& \alpha_{\mathrm{p}} \gg\left(\alpha_{\theta}+\gamma_{2} \cdot \bar{N}+\lambda\right), \quad \omega^{2} \gg \lambda^{2} \\
& \hat{s}_{N N}(f)=\frac{\alpha_{\mathrm{t}} \cdot \bar{N}}{\left(\omega^{2}+\alpha_{\mathrm{p}}^{2}\right)} \\
& \widehat{s}_{N N}(f) \cong \frac{\overline{v \cdot(v-1) \cdot F_{\mathrm{r}}}}{\left[\omega^{2}+[(\rho-\beta) / \operatorname{tg}]^{2}\right]} \text { pour } f>1
\end{aligned}
$$

avec :

$$
F_{\mathrm{r}}=\frac{\bar{N} \cdot k_{\mathrm{e}}}{\bar{v} \cdot l}
$$

nombre de fissions dans le réacteur par seconde.

$\widehat{s}_{N N}(f)$ peut donc être représenté par le module au carré de la fonction de transfert d'un filtre passe-bas $\mathrm{du}$ premier ordre, ayant une fréquence de coupure

$$
f_{N}=-\frac{\rho-\beta}{2 \pi \operatorname{tg}} .
$$

Remarques. - 1. Pour un réacteur critique $(\rho=0)$, nous déterminons bien $f_{N}=\beta / 2 \pi l=\alpha_{\mathrm{p}_{0}} / 2 \pi$ et non pas $f_{N}^{\prime}=1 / 2 \pi \cdot\left[\alpha_{\mathrm{p}_{0}}+\gamma_{\mathrm{u}} \cdot\left(\bar{\theta}_{\mathrm{u}}-\theta_{\mathrm{u}_{0}}\right)\right]$, avec

$$
\alpha_{\mathrm{p}_{0}}=-\alpha_{\mathrm{f}_{0}} \cdot\left(\bar{v}_{0}-1\right)+\alpha_{\mathrm{l}_{0}},
$$

car le terme $\gamma_{u} \cdot\left(\bar{\theta}_{u}-\theta_{u_{0}}\right)$, positif $\left(\gamma_{u}>0\right)$, rendrait le réacteur sous-critique lors d'une élévation de puissance, est exactement compensé par le déplacement des barres de contrôle. La fréquence de coupure de la densité spectrale énergétique neutronique reste donc la même quelle que soit la puissance.

2. D'après la remarque 1 , nous déduisons que la contre-réaction due à la température du combustible n'influence pas la fréquence de coupure de la densité spectrale énergétique neutronique.

3. Cette fréquence de coupure pourrait dépendre du niveau de puissance, donc du coefficient de réactivité $\gamma_{\mathrm{u}}$, dans le cas de contre-réaction de température, si au lieu de compenser le terme $\gamma_{u} \cdot\left(\ldots \bar{\theta}_{u}-\theta_{u_{0}}\right)$ par un déplacement des barres de contrôle, on le compensait par une source extérieure $\bar{S}$ telle que la population totale des neutrons reste la même. Ceci n'est malheureusement pas réalisable pratiquement pour des puissances supérieures à quelques centièmes de watt.

3.2 Densité SPECTRALE ÉNERGÉTIQUE THERMIQUE. - Le dernier élément diagonal de la matrice $S(f)$ donne la densité spectrale énergétique des fluctuations de la température du combustible, ou plus exactement celle des fluctuations du nombre d'unités d'énergie interne contenues dans le combustible :

$$
\begin{aligned}
s_{\theta_{\mathrm{u}} \theta_{\mathrm{u}}}(f)= & \frac{\alpha_{\mathrm{f}} \cdot<N>}{a^{2}+\omega^{2} \cdot b^{2}} \cdot\left\{\left(\omega^{2}+\lambda^{2}\right) \cdot\left[\alpha_{\mathrm{t}} \cdot \alpha_{\mathrm{f}}+2 \alpha_{\mathrm{f}} \cdot \alpha_{\mathrm{p}} \cdot\left(<v_{0}>-1\right)+2 \cdot\left(\omega^{2}+\alpha_{\mathrm{p}}^{2}\right)\right]+\right. \\
& +\lambda^{2} \cdot \alpha_{\mathrm{f}}^{2} \cdot\left[\beta \cdot<v \cdot(\beta \cdot<v>+1)>+2 \beta \cdot<v \cdot\left(v_{0}-2\right)>-2 \beta \cdot<v>\cdot\left(<v_{0}>-1\right)\right] \\
& \left.+2 \beta \cdot<v>\cdot \lambda \cdot \alpha_{\mathrm{f}} \cdot\left(\omega^{2}-\lambda \cdot \alpha_{\mathrm{p}}\right)\right\} \\
& +\frac{2 \alpha_{\mathrm{m}} \cdot \theta_{\mathrm{m}_{0}}}{a^{2}+\omega^{2} \cdot b^{2}} \cdot\left[\left(\omega^{2}+\alpha_{\mathrm{p}}^{2}\right) \cdot\left(\omega^{2}+\lambda^{2}\right)+2 \beta \cdot<v>\cdot \lambda \cdot \alpha_{\mathrm{f}} \cdot\left(\omega^{2}-\lambda \cdot \alpha_{\mathrm{p}}\right)\right. \\
& \left.+(\beta \cdot<v>)^{2} \cdot \lambda^{2} \cdot \alpha_{\mathrm{f}}^{2}\right]
\end{aligned}
$$

avec $a$ et $b$ définis par (15) et (16). 
Si le coefficient d'échange thermique $\gamma$ est faible, c'est-à-dire que nous sommes dans le cas de la convection naturelle, $\mathrm{s}_{\theta_{\mathrm{u}} \theta_{\mathrm{u}}}(f)$ est proportionnelle à $\langle N\rangle$. Pour $\gamma$ grand, cas de la convection forcée, la densité spectrale énergétique thermique ne reste proportionnelle à $\langle N\rangle$ que pour des fréquences inférieures à $10^{-3} \mathrm{~Hz}$. Une augmentation du coefficient d'échange thermique entraîne une diminution de l'amplitude des fluctuations de la température du combustible. Pour les basses fréquences $s_{\theta_{\mathrm{u}} \theta_{\mathrm{u}}}(f)$ est proportionnelle à $1 / \gamma^{2}$ et pour les moyennes à $1 / \gamma$.

La figure 3 représente la densité spectrale énergétique thermique pour, différents régimes de refroidissement (convection naturelle, forcée laminaire et turbulent), pour un réacteur fonctionnant à une centaine de watts et ayant un coefficient de température du combustible de $-2,5 \mathrm{pcm} /{ }^{\circ} \mathrm{K}$.

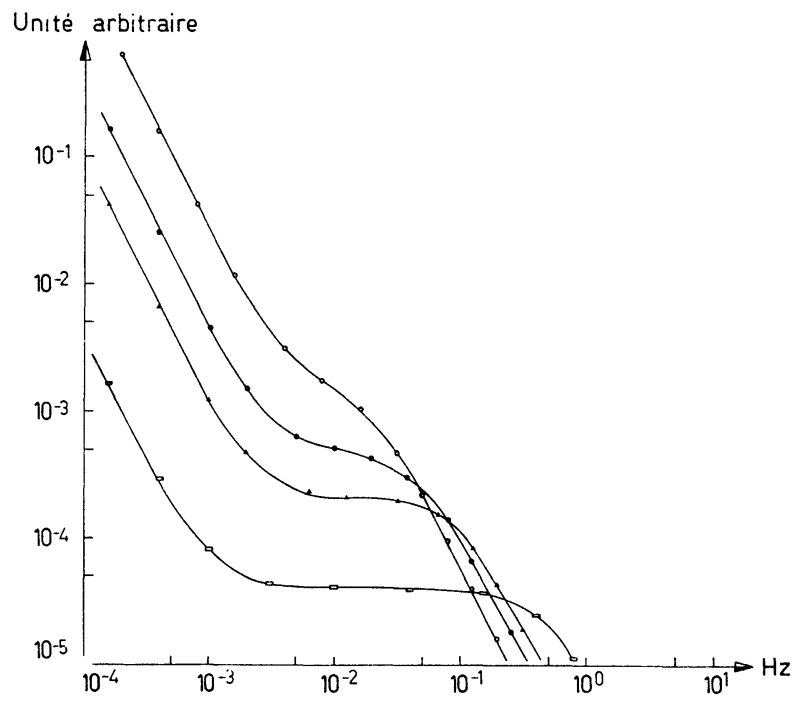

FIG. 3.

$\langle N\rangle=1 \times 10^{9}\left(P_{\text {react }} \cong 100 \mathrm{~W}\right)$

$l=65 \mu \mathrm{s}$

$\beta=800 \mathrm{pcm}$

$\lambda=0,05 \mathrm{rd} / \mathrm{s}$

$\partial \rho / \partial T_{\mathrm{u}}=-2,5 \mathrm{pcm} /{ }^{\circ} \mathrm{K}$

-O- $\gamma=200 \mathrm{~W} / \mathrm{m}^{2} \cdot{ }^{\circ} \mathrm{K}$

$-\gamma=500 \mathrm{~W} / \mathrm{m}^{2} .{ }^{\circ} \mathrm{K}$

- $-\gamma=1000 \mathrm{~W} / \mathrm{m}^{2} \cdot{ }^{\circ} \mathrm{K}$

Pour des puissances de fonctionnement de l'ordre de quelques kilowatts, une augmentation du coefficient de température du combustible, s'il est négatif, peut se traduire par une diminution importante de l'amplitude des fluctuations de la température du combustible.

En supposant à nouveau que l'hypothèse d'ergodicité soit applicable et compte tenu des relations (17) à (22), l'expression (26) peut se simplifier en considérant des fréquences $f<1$ pour un réacteur voisin de la criticité ou critique

$$
\begin{aligned}
\hat{s}_{\theta_{\mathrm{u}} \theta_{\mathrm{u}}}(f)= & \frac{\alpha_{\mathrm{f}}^{2} \cdot \alpha_{\mathrm{t}} \cdot \bar{N}+2 \alpha_{\mathrm{m}} \cdot \theta_{\mathrm{m}_{0}} \cdot \alpha_{\mathrm{p}}^{2}}{\alpha_{\mathrm{p}}^{2}} \times \\
& \left.\times \frac{\left(\omega^{2}+\lambda^{2} \cdot \frac{\alpha_{\mathrm{f}}^{2} \cdot \alpha_{\mathrm{t}} \cdot \bar{N}}{\alpha_{\mathrm{f}}^{2} \cdot \alpha_{\mathrm{t}} \cdot \bar{N}+2}\right.}{\omega \cdot{ }^{2}\left(\omega^{2}+\alpha_{\theta}^{2}\right)}\right)
\end{aligned}
$$

soit :

$$
\widehat{s}_{\theta_{\mathrm{u}} \theta_{\mathrm{u}}}(f)=\chi^{*} \cdot \frac{\left(\omega^{2}+\hat{\lambda}_{\mathrm{eq}}^{2}\right)}{\omega^{2} \cdot\left(\omega^{2}+\alpha_{\theta}^{2}\right)}
$$

avec :

$$
\begin{gathered}
\chi^{*}=\frac{\overline{v \cdot(v-1)} \cdot F_{\mathrm{r}}}{\beta^{2} \cdot \bar{v}^{2}}+\frac{2 \gamma \cdot S_{\mathrm{e}} \cdot T_{\mathrm{m}_{\mathrm{o}}}}{q} \\
\lambda_{\mathrm{eq}}^{2}=\lambda^{2} \cdot \frac{\overline{v \cdot(v-1)} \cdot F_{\mathrm{r}}}{\beta^{2} \cdot \bar{v}^{2}} \cdot \frac{1}{\chi^{*}} .
\end{gathered}
$$

Lorsque la puissance du réacteur augmente, le premier terme du membre de droite de l'expression (29) devient plus important que le second, en conséquence $\lambda_{\text {eq }}$ tend vers $\lambda$, et l'expression (27) vers :

$$
\hat{s}_{\theta_{\mathrm{u}} \theta_{\mathrm{u}}}(f)=\frac{\overline{v \cdot(v-1)}}{\beta^{2} \cdot \bar{v}^{2}} \cdot F_{\mathrm{r}} \cdot \frac{\left(\omega^{2}+\lambda^{2}\right)}{\omega^{2} \cdot\left(\omega^{2}+\alpha_{\theta}^{2}\right)} .
$$

Remarque. - En réalité, le dénominateur de (28) ou (31) n'est pas $\beta^{2} \cdot \bar{v}^{2} \cdot \omega^{2} \cdot\left(\omega^{2}+\alpha_{\theta}^{2}\right)$ mais

$$
\beta^{2} \cdot \bar{v}^{2} \cdot \omega^{2} \cdot\left[\omega^{2}+\left(\alpha_{\theta}+\gamma_{2} \cdot \bar{N}\right)^{2}\right],
$$

$\gamma_{2}$ étant comme nous l'avons vu précédemment, le coefficient de température agissant sur la probabilité de fission. Toutefois, nous avons généralement $\alpha_{\theta} \ll \gamma_{2} \cdot \bar{N}$ pour des réacteurs fonctionnant à des puissances inférieures à $100 \mathrm{~kW}$ et ayant un coefficient de température du combustible (effet Doppler, effet de densité et de fuite) de quelques $\mathrm{pcm} /{ }^{\circ} \mathrm{K}$. La contre-réaction n'influence donc pas la fréquence de coupure $f_{\theta}=\alpha_{\theta} / 2 \pi$ de $\hat{s}_{\theta_{\mathrm{u}} \theta_{\mathrm{u}}}(f)$.

3.3. DENSITÉ SPECTRALE D'INTERACTION NEUTRONSTEMPÉRATURE DU COMBUSTIBLE. - L'élément de la première ligne, troisième colonne de la matrice $S(f)$ donne la densité spectrale d'interaction neutronstempérature du combustible, ou plus exactement la densité spectrale d'interaction neutrons-nombre d'unités d'énergie interne contenues dans le combustible :

$$
s_{N \theta_{u}}(f)=\frac{A+i \omega \cdot B}{a^{2}+\omega^{2} \cdot b^{2}}
$$

avec :

$$
\begin{aligned}
& A=\alpha_{\mathrm{f}} \cdot\langle N\rangle \cdot\left\{( \omega ^ { 2 } + \lambda ^ { 2 } ) \cdot \left[( < v _ { 0 } > - 1 ) \cdot \left[\omega^{2}+\alpha_{\mathrm{p}} \cdot\left(\alpha_{\theta}+\gamma_{2} \cdot\langle N>)\right]+\alpha_{\mathrm{t}} \cdot\left(\alpha_{\theta}+\gamma_{2} \cdot\langle N>)-\right.\right.\right.\right. \\
& -\left(\left\langle v_{0}>-1\right) \cdot \alpha_{\mathrm{f}} \cdot \gamma_{\mathrm{u}} \cdot\langle N\rangle+2 \alpha_{\mathrm{p}} \cdot \gamma_{\mathrm{u}} \cdot\langle N>]\right. \\
& +\beta \cdot\left\langle v>\cdot \lambda \cdot\left[-\lambda \cdot \gamma_{2} \cdot\left[( < v _ { 0 } > - 1 ) \cdot \alpha _ { \mathrm { f } } \cdot \left\langleN>-\beta \cdot\left\langle v>\cdot \alpha_{\mathrm{f}} \cdot\langle N>]\right.\right.\right.\right.\right. \\
& +\left(<v_{0}>-1\right) \cdot \alpha_{\mathrm{f}} \cdot\left[\omega^{2}-\lambda \cdot\left(\alpha_{\theta}+\gamma_{2} \cdot<N>\right)\right]+\alpha_{\mathrm{p}} \cdot \lambda \cdot\left(\alpha_{\theta}+\gamma_{2} \cdot<N>\right) \\
& +\omega^{2} \cdot\left[\alpha_{\mathrm{p}}+\lambda-\left(\alpha_{\theta}+\gamma_{2} \cdot\langle N>)\right]+\alpha_{\mathrm{f}} \cdot \lambda \cdot \gamma_{\mathrm{u}} \cdot\left\langle N>+2 \gamma_{2} \cdot\langle N\rangle \cdot\left(\omega^{2}-\lambda \cdot \alpha_{\mathrm{p}}\right)\right]\right. \\
& +\alpha_{\mathrm{f}} \cdot \lambda^{2} \cdot\left(\alpha_{\theta}+\gamma_{2} \cdot<N>\right) \cdot\left[\beta \cdot\left\langle v \cdot(\beta \cdot<v>+1)>+2 \beta \cdot<v \cdot\left(v_{0}-2\right)>-(\beta \cdot<v>)^{2}\right]\right\} \\
& +2 \alpha_{\mathrm{m}} \cdot \theta_{\mathrm{m}_{0}} \cdot\left\langle N>\cdot\left[\alpha_{\mathrm{p}} \cdot \gamma_{\mathrm{u}} \cdot\left(\omega^{2}+\lambda^{2}\right)+\left(\beta \cdot\langle v>)^{2} \cdot \alpha_{\mathrm{f}} \cdot \lambda^{2} \cdot \gamma_{2}+\beta \cdot\left\langle v>\cdot \alpha_{\mathrm{f}} \cdot \lambda^{2} \cdot \gamma_{\mathrm{u}}\right.\right.\right.\right. \\
& +\beta \cdot\left\langle v>\cdot \lambda \cdot \gamma_{2} \cdot\left(\omega^{2}-\lambda \cdot \alpha_{\mathrm{p}}\right)\right]
\end{aligned}
$$




$$
\begin{aligned}
B= & \alpha_{\mathrm{f}} \cdot\left\langle N>\cdot\left\{( \omega ^ { 2 } + \lambda ^ { 2 } ) \cdot \left[( < v _ { 0 } > - 1 ) \cdot \left[\alpha_{\mathrm{p}}-\left(\alpha_{\theta}+\gamma_{2} \cdot\langle N>)\right]+\alpha_{\mathrm{t}}+2 \gamma_{\mathrm{u}} \cdot\langle N>]+\right.\right.\right.\right. \\
& +\beta \cdot<v>\cdot \lambda \cdot\left[\left(<v_{0}>-1\right) \cdot \alpha_{\mathrm{f}} \cdot \gamma_{2} \cdot<N>+\alpha_{\mathrm{f}} \cdot \gamma_{\mathrm{u}} \cdot\left\langle N>+2 \gamma_{2} \cdot\left\langle N>\cdot\left(\alpha_{\mathrm{p}}+\lambda\right)\right.\right.\right. \\
& -\left(<v_{0}>-1\right) \cdot \alpha_{\mathrm{f}} \cdot\left[\lambda+\left(\alpha_{\theta}+\gamma_{2} \cdot<N>\right)\right]-\beta \cdot<v>\cdot \alpha_{\mathrm{f}} \cdot \lambda-\left[\omega^{2}+\left(\alpha_{\mathrm{p}}+\lambda\right) \cdot\left(\alpha_{\theta}+\gamma_{2} \cdot<N>\right)\right. \\
& \left.\left.\left.-\alpha_{\mathrm{p}} \cdot \lambda\right]\right]+\alpha_{\mathrm{f}} \cdot \lambda^{2} \cdot\left[\beta \cdot<v \cdot(\beta \cdot<v>+1)>+2 \beta \cdot<v \cdot\left(v_{0}-2\right)>\right]\right\} \\
& +2 \alpha_{\mathrm{m}} \cdot \theta_{\mathrm{m}_{0}} \cdot\left\langle N>\cdot\left[\gamma_{\mathrm{u}} \cdot\left(\omega^{2}+\lambda^{2}\right)+\beta \cdot<v>\cdot \lambda \cdot \gamma_{2} \cdot\left(\alpha_{\mathrm{p}}+\lambda\right)+\beta \cdot\left\langle v>\cdot \alpha_{\mathrm{f}} \cdot \lambda \cdot \gamma_{\mathrm{u}}\right] .\right.\right.
\end{aligned}
$$

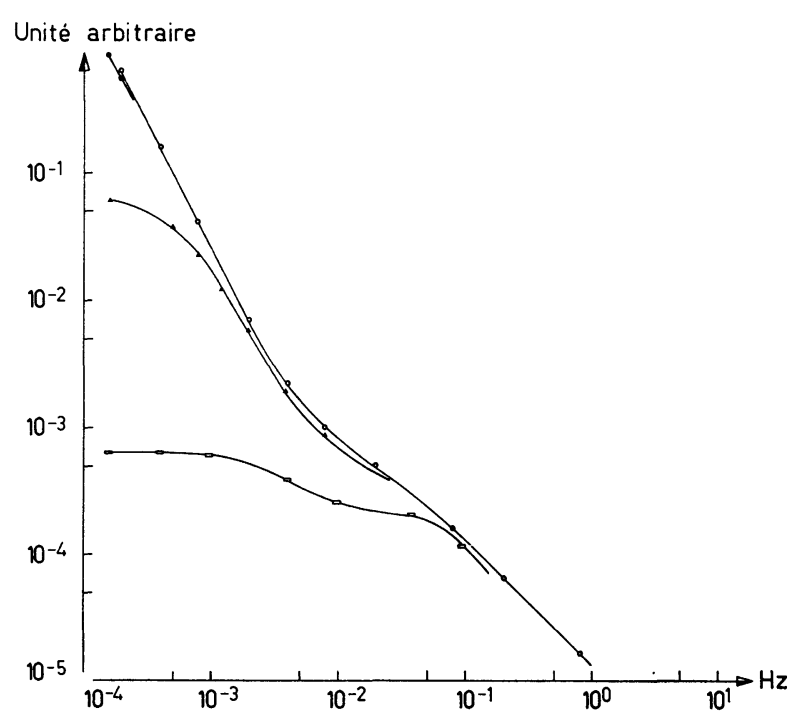

FIG. $4 a$.

$$
\begin{gathered}
<N>=2,5 \times 10^{11}\left(P_{\text {react }} \cong 50 \mathrm{~kW}\right) \\
l=65 \mu \mathrm{s} \\
\beta=800 \mathrm{pcm} \\
\lambda=0,05 \mathrm{rd} / \mathrm{s} \\
\gamma=200 \mathrm{~W} / \mathrm{m}^{2}{ }^{\circ} \mathrm{K} \\
-0-\quad \partial \rho / \partial T_{\mathbf{u}}=-0,1 \mathrm{pcm} /{ }^{\circ} \mathrm{K} \\
-10 \mathrm{pcm} /{ }^{\circ} \mathrm{K} \\
-\triangle-\quad \partial \rho / \partial T_{\mathbf{u}}=-10 \mathrm{pcm} /{ }^{\circ} \mathrm{K} \\
-\square-\quad \partial \rho / \partial T_{\mathbf{u}}=-100 \mathrm{pcm} /{ }^{\circ} \mathrm{K}
\end{gathered}
$$

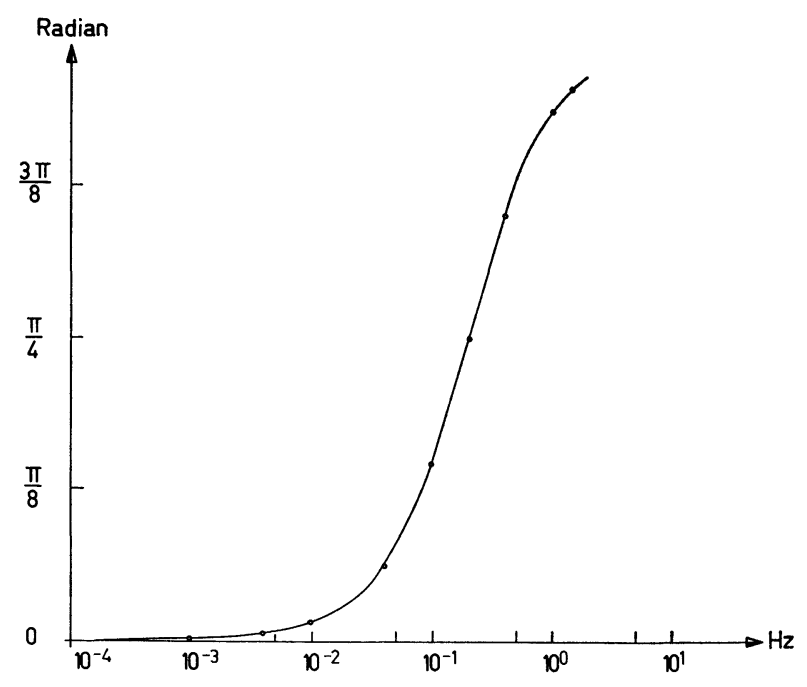

FIG. $4 b$.

$<N>=2,5 \times 10^{11}\left(P_{\text {react }} \cong 50 \mathrm{~kW}\right)$

$l=65 \mu \mathrm{s}$

$\beta=800 \mathrm{pcm}$

$\lambda=0,05 \mathrm{rd} / \mathrm{s}$

$\gamma=200 \mathrm{~W} / \mathrm{m}^{2} \quad \mathrm{~K}$

$\partial \rho / \partial T_{\mathrm{u}}=-0,1$ à $-100 \mathrm{pcm} /{ }^{\circ} \mathrm{K}$
Le module de $s_{N \theta_{u}}(f)$ est proportionnel à $\langle N\rangle$. Le coefficient de température du combustible a le même effet sur $s_{N \theta_{\mathrm{u}}}(f)$ que sur $s_{\theta_{\mathrm{u}} \theta_{\mathrm{u}}}(f)$. Par contre, l'argument de $s_{N \theta_{\mathrm{u}}}(f)$ n'est pas affecté par ce coefficient de température.

Les figures $4 a$ et $4 b$ représentent respectivement le module et l'argument de la densité spectrale d'interaction neutrons-température du combustible, pour différents coefficients de température et pour un réacteur refroidi en convection naturelle, fonctionnant à environ $50 \mathrm{~kW}$.

Nous remarquons également que le module de $s_{N \theta_{\mathrm{u}}}(f)$ occupe à peu près le même domaine fréquentiel que $s_{\theta_{\mathrm{u}} \theta_{\mathrm{u}}}(f)$.

L'hypothèse d'ergodicité et les relations (17) à (22) nous permettent de simplifier l'expression (32), puis d'en calculer le module et l'argument pour des fréquences $f<1$ pour un réacteur voisin de la criticité ou critique

$$
\begin{aligned}
& \hat{s}_{N \theta_{\mathrm{u}}}(f)= \\
& =\frac{\sqrt{\left[\alpha_{\mathrm{f}} \cdot \alpha_{\mathrm{t}} \cdot \alpha_{\theta} \cdot \bar{N} \cdot\left(\omega^{2}+\lambda^{2}\right)\right]^{2}+\left[\omega \cdot \alpha_{\mathrm{f}} \cdot \alpha_{\mathrm{t}} \cdot \bar{N} \cdot\left(\omega^{2}+\lambda^{2}\right)\right]^{2}}}{\alpha_{\mathrm{p}}^{2} \cdot \omega^{2} \cdot\left(\omega^{2}+\alpha_{\theta}^{2}\right)}
\end{aligned}
$$

car :

$\alpha_{t}, \alpha_{\theta} \gg$ aux autres termes du membre de droite de (33),

$\alpha_{t} \gg \quad$ aux autres termes du membre de droite de$$
\alpha_{\theta} \gg \quad \gamma_{2} \cdot \bar{N},
$$

soit :

$$
\widehat{s}_{N \theta_{\mathrm{u}}}(f)=\frac{\overline{v \cdot(v-1)}}{\beta^{2} \cdot \bar{v}^{2}} \cdot F_{\mathrm{r}} \cdot \frac{\left(\omega^{2}+\lambda^{2}\right)}{\omega^{2} \cdot \sqrt{\omega^{2}+\alpha_{\theta}^{2}}} .
$$

En plus, compte tenu des simplifications intervenues dans la partie réelle et la partie imaginaire de la densité spectrale d'interaction neutrons-température du combustible, l'argument de cette dernière s'écrit :

$$
\widehat{\Psi}_{N \theta_{\mathrm{u}}}=\operatorname{arctg}\left(\frac{\omega}{\alpha_{\theta}}\right) \text {. }
$$

Pour les mêmes raisons que celles invoquées pour la densité spectrale énergétique thermique, la contreréaction de température du combustible n'influence pas la fréquence de coupure de $s_{N \theta_{\mathrm{u}}}(f)$.

4. Schéma équivalent. Détermination des paramètres du réacteur. - Le comportement du réac- 
teur, compte tenu de l'influence de la température du combustible sur les propriétés nucléaires de ce der- nier, peut se schématiser pour de petites variations par le système bouclé suivant :

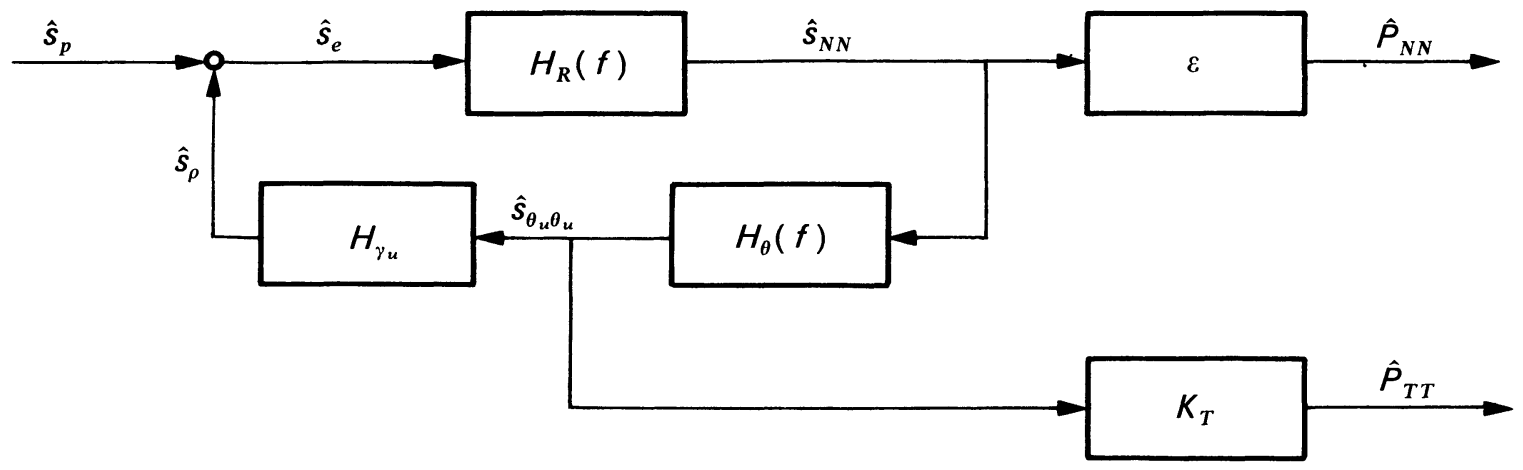

\begin{abstract}
$H_{\mathbf{R}}(f)$
fonction de transfert du réacteur (fréquence de coupure $f_{N}$ ),

$H_{\theta}(f)$ fonction de transfert reliant les fluctuations de la population neutronique à celles de la température du combustible (fréquence de coupure $f_{\theta}$ ),

$H_{\gamma_{\mathrm{u}}} \quad$ fonction de transfert reliant les fluctuations de température à celles de réactivité (proportionnelle au coefficient de température du combustible),

$\varepsilon \quad$ fonction de transfert reliant les fluctuations de la population neutronique à celles apparaissant à la sortie d'un détecteur (efficacité du détecteur),

$K_{T} \quad$ fonction de transfert reliant les fluctuations de la température du combustible à celles apparaissant à la sortie de l'appareil de mesure,
\end{abstract}

$\hat{s}_{\mathrm{p}} \quad$ densité spectrale énergétique du bruit

propre en réactivité du réacteur,

$\widehat{s}_{\mathrm{e}}=\widehat{s}_{\mathrm{p}}+\widehat{s}_{\rho}$ densité spectrale énergétique à l'entrée du réacteur,

$\hat{s}_{\rho}$

densité spectrale énergétique des fluctuations de réactivité dues aux fluctuations de la température du combustible, $\widehat{s}_{N N} \quad$ densité spectrale énergétique neutronique,

$\widehat{P}_{\mathrm{NN}} \quad$ estimation de la densité spectrale énergétique neutronique (densité spectrale énergétique des fluctuations à la sortie d'un détecteur),

$\widehat{s}_{\theta_{\mathbf{u}} \theta_{\mathrm{u}}} \quad$ densité spectrale énergétique thermique des fluctuations de la température du combustible dues à celles de la population neutronique,

$\widehat{P}_{\text {TT }} \quad$ estimation de la densité spectrale énergétique thermique (densité spectrale énergétique des fluctuations à la sortie de l'appareil de mesure).

Le schéma équivalent ci-dessus et les expressions (25), (28) ou (31), (36) ou (37) nous permettent de calculer certains paramètres du réacteur.
La densité spectrale énergétique neutronique $\widehat{s}_{N N}(f)$ permet de déterminer la fréquence de coupure $f_{N}=\beta / 2 \pi l$ pour un réacteur critique, donc de calculer le temps de vie moyen des neutrons $l$, en supposant connue la fraction totale des neutrons retardés.

La densité spectrale énergétique thermique $\hat{s}_{\theta_{\mathrm{u}} \theta_{\mathrm{u}}}(f)$ permet de déterminer la fréquence de coupure $f_{\theta}=\gamma S_{\mathrm{e}} / 2 \pi C_{\mathrm{u}}$ et d'en déduire le coefficient d'échange thermique $\gamma$ en se donnant $S_{\mathrm{e}}$ et $C_{\mathrm{u}}$.

A partir de $\hat{s}_{\theta_{\mathbf{u}} \theta_{\mathbf{u}}}(f)$ nous pouvons également évaluer la constante de décroissance des précurseurs $\lambda$. Ceci est facilité car $\lambda$ est du même ordre de grandeur que $\alpha_{\theta}$. Ces deux paramètres $\gamma$ et $\lambda$ peuvent également être calculés à partir de la densité spectrale d'interaction neutrons-température du combustible $\widehat{s}_{N \theta_{u}}(f)$.

Comme nous le verrons dans la seconde partie de ce travail, les densités spectrales énergétiques neutronique et thermique seront estimées respectivement par $\widehat{P}_{\mathrm{NN}}(f)$ et $\widehat{P}_{\mathrm{TT}}(f)$. Nous aurons donc la possibilité d'obtenir expérimentalement le temps de vie moyen des neutrons, le coefficient d'échange thermique et la constante de décroissance des précurseurs.

5. Conclusions. - L'analyse probabiliste du comportement du réacteur nous a permis d'une part de confirmer les résultats déjà obtenus par D. R. Harris et P. R. Pluta, pour les valeurs moyennes et les covariances ; d'autre part d'exprimer les densités spectrales relatives aux fluctuations de la population des neutrons et de la température du combustible.

Pour ces dernières, nous pouvons définir deux domaines distincts :

- de $10^{-4} \mathrm{~Hz}$ à $10^{-1} \mathrm{~Hz}$ : domaine dans lequel les effets thermiques jouent un grand rôle [10], notamment le coefficient de convection entre le combustible et le réfrigérant et le coefficient de température du combustible. Ce dernier, s'il est grand et négatif, peut diminuer notablement tant les fluctuations de la population neutronique que les fluctuations de la température du combustible ;

- au-delà de $10^{-1} \mathrm{~Hz}$ : domaine dans lequel les effets thermiques ne jouent aucun rôle. La densité 
spectrale énergétique neutronique n'est pas modifiée et notamment le facteur $\beta / l$.

Les densités spectrales ci-dessus citées peuvent se simplifier en considérant des gammes de fréquence bien choisies ; ceci a conduit à la mise en œuvre d'une méthode expérimentale de mesures du facteur $\beta / l$ et du coefficient d'échange thermique $\gamma$. Cette méthode sera décrite dans la seconde partie.

Une extension du modèle au cas de plusieurs chaînes de contre-réactions, par exemple dues aux effets de températures du réfrigérant et du combustible, est possible. Nous pourrions ainsi exprimer la densité spectrale énergétique relative aux fluctuations de la température du réfrigérant.

Annexe A. - L'hypothèse 2 permet de dire que le comportement du réacteur peut être caractérisé par un processus markovien continu dans l'espace du vecteur d'état $\mathbf{y}$ et du temps.

Soit maintenant

$$
p\left(N, C, \theta_{\mathrm{u}}, t \mid N_{0}, C_{0}, \theta_{\mathrm{u}_{0}}, t_{0}\right) \cdot \mathrm{d} N \cdot \mathrm{d} C \cdot \mathrm{d} \theta_{\mathrm{u}}
$$

la probabilité conditionnelle pour que la population des neutrons soit comprise entre $N$ et $N+\mathrm{d} N$, celle des précurseurs entre $C$ et $C+\mathrm{d} C, \ldots$, au temps $t$, étant donné qu'au temps $t_{0}$ on avait $N_{0}$ neutrons, $C_{0}$ précurseurs...

Nous pouvons alors développer l'équation de Chapman-Kolmogoroff en série de Taylor autour de $N, C, \theta_{\mathrm{u}}$ et ne garder que les deux premiers termes. Nous obtenons ainsi l'équation de FokkerPlanck :

$$
\begin{array}{r}
\frac{\delta p\left(N, C, \theta_{\mathrm{u}}, t \mid N_{0}, C_{0}, \theta_{\mathrm{u}_{0}}, t_{0}\right)}{\delta t}=\sum_{k=0}^{M} \frac{\delta}{\delta y_{k}}\left[\frac{1}{2} \cdot \sum_{l=0}^{M} \frac{\delta}{\delta y_{l}} \sigma_{k l}\left(N, C, \theta_{\mathrm{u}}, t\right) \cdot p\left(N, C, \theta_{\mathrm{u}}, t \mid N_{0}, C_{0}, \theta_{\mathrm{u}_{0}}, t_{0}\right)\right]- \\
-\quad-m_{k}\left(N, C, \theta_{\mathrm{u}}, t\right) \cdot p\left(N, C, \theta_{\mathrm{u}}, t \mid N_{0}, C_{0}, \theta_{\mathrm{u}_{0}}, t_{0}\right)
\end{array}
$$

avec :

$$
\begin{aligned}
& m_{0}(\mathbf{y}, t)=\left[\alpha_{\mathrm{f}}(t) \cdot\left(\left\langle v_{0}\right\rangle-1\right)-\alpha_{l}(t)\right] \cdot N+\lambda \cdot C+S(t) \\
& m_{1}(\mathbf{y}, t)=\alpha_{\mathrm{f}}(t) .<v_{i}>. N-\lambda . C \\
& m_{\theta_{\mathrm{u}}}(\mathbf{y}, t)=\alpha_{\mathrm{f}}(t) \cdot N-\alpha_{\theta}(t) \cdot \theta_{\mathrm{u}}+\alpha_{\mathrm{m}}(t) \cdot \theta_{\mathrm{m}_{0}} \\
& \sigma_{00}(\mathbf{y}, t)=\left[\alpha_{\mathrm{f}}(t) \cdot\left\langle v_{0}-1>^{2}+\alpha_{l}(t)\right] \cdot N+\lambda \cdot C+S(t)\right. \\
& \sigma_{0_{\mathbf{i}}}(\mathbf{y}, t)=\sigma_{\mathrm{i}_{0}}(\mathbf{y}, t)=\left\langle\left(v_{0}-1\right) \cdot v_{\mathrm{i}}\right\rangle \cdot \alpha_{\mathrm{f}}(t) \cdot N-\lambda \cdot C \\
& \sigma_{\mathrm{ii}}(\mathbf{y}, t)=\left\langle v_{\mathrm{i}}^{2}>\cdot \alpha_{\mathrm{f}}(t) \cdot N+\lambda \cdot C\right. \\
& \sigma_{0_{0_{\mathbf{u}}}}(\mathbf{y}, t)=\sigma_{\theta_{\mathbf{u}_{0}}}(\mathbf{y}, t)=\left(\left\langle v_{0}>-1\right) \cdot \alpha_{\mathrm{f}}(t) \cdot N\right. \\
& \sigma_{\mathbf{i}_{0_{\mathrm{u}}}}(\mathbf{y}, t)=\sigma_{\theta_{\mathbf{u}_{\mathrm{i}}}}(\mathbf{y}, t)=\left\langle v_{\mathrm{i}}\right\rangle \cdot \alpha_{\mathrm{f}}(t) \cdot N \\
& \sigma_{\theta_{\mathrm{u}} \theta_{\mathrm{u}}}(\mathbf{y}, t)=\alpha_{\mathrm{f}}(t) \cdot N+\alpha_{\theta}(t) \cdot \theta_{\mathrm{u}}+\alpha_{\mathrm{m}}(t) \cdot \theta_{\mathrm{m}_{0}} \cdot
\end{aligned}
$$

Remarque. - La densité de probabilité conditionnelle doit être positive, son intégrale doit être égale à 1 (normalisation) et pour $t \rightarrow t_{0}$ :

$$
\lim _{t \rightarrow t_{0}} p\left(\mathbf{y}, t \mid \mathbf{y}_{0}, t_{0}\right)=\delta\left(\mathbf{y}-\mathbf{y}_{0}\right)
$$

avec $\delta\left(\mathbf{y}-\mathbf{y}_{0}\right)$ fonction delta de Dirac.

Nous pouvons montrer qu'en partant des définitions des moments du premier, du deuxième ordre, et de l'équation de Fokker-Planck, nous avons [1], [2] :

$$
\begin{gathered}
\frac{\mathrm{d}}{\mathrm{d} t}<y_{k}(t)>=<m_{k}(\mathbf{y}, t)>\quad \text { pour } \quad k=N, C, \theta_{\mathrm{u}} \\
\frac{\mathrm{d}}{\mathrm{d} t} \mu_{k l}(t)=<\left[y_{k}(t)-<y_{k}(t)>\right] \cdot\left[m_{l}(\mathbf{y}, t)-\left\langle m_{l}(\mathbf{y}, t)>\right]>+\right. \\
+<\left[y_{l}(t)-\left\langle y_{l}(t)>\right] \cdot\left[m_{k}(\mathbf{y}, t)-\left\langle m_{k}(\mathbf{y}, t)>\right]>+<\sigma_{k l}(\mathbf{y}, t)>\right.\right.
\end{gathered}
$$

avec $t>t_{0}$ et pour $k, l=N, C, \theta_{\mathrm{u}} ; \mu_{k l}(t)$ : covariance. 
D'une manière analogue, nous pouvons montrer qu'en partant de la définition des fonctions de corrélation et de l'équation de Fokker-Planck écrite avec les densités de " probabilité jointe " $p\left(\mathbf{y}^{1}, t_{1} ; \mathbf{y}^{2}, t_{2}\right)$, nous avons [2] :

$$
\begin{aligned}
& \frac{\mathrm{d}}{\mathrm{d} t_{2}} \varphi_{k l}\left(t_{1}, t_{2}\right)=<\left[y_{k}\left(t_{1}\right)-<y_{k}\left(t_{1}\right)>\right] \times \\
& \times\left[m_{l}\left(\mathbf{y}, t_{2}\right)-<m_{l}\left(\mathbf{y}, t_{2}\right)>\right]>
\end{aligned}
$$

avec $t_{1}<t_{2}$ et pour $k, l=N, C, \theta_{\mathrm{u}} ; \varphi_{k l}\left(t_{1}, t_{2}\right)$ : fonction de corrélation.

(A7) doit être résolue avec la condition aux limites :

$$
\lim _{t_{2} \rightarrow t_{1}} \varphi_{k l}\left(t_{1}, t_{2}\right)=\mu_{k l}\left(t_{1}\right)
$$

$\mu_{k l}\left(t_{1}\right)$ étant la solution de (A6) avec $t_{1}>t_{0}$.

Annexe B. - Un processus aléatoire markovien et gaussien satisfait l'équation de Fokker-Planck linéarisée (théorème de Doob généralisée) [9].

Nous pouvons alors écrire le système (A5), compte tenu de (A2) et (11) :

$$
\frac{\mathrm{d}}{\mathrm{d} t}<\Delta \mathbf{y}(t)>=\Lambda \cdot\langle\Delta \mathbf{y}(t)>
$$

avec :

$$
y_{k}(t)=\left\langle y_{k}(t)\right\rangle+\Delta y_{k}(t)
$$

$\Delta y_{k}(t)$ variable aléatoire instantanée du processus, $\left(\Delta y_{k}(t)\right.$ petit par rapport à $\left.\left\langle y_{k}(t)\right\rangle\right)$.

La méthode de Langevin consiste à écrire (B1) sous la forme suivante :

$$
\frac{\mathrm{d}}{\mathrm{d} t} \Delta \mathbf{y}(t)+\Lambda^{\prime} \cdot \Delta \mathbf{y}(t)=\mathbf{F}(t)
$$

avec :

$$
\begin{aligned}
& <\mathbf{F}(t)>=0 \\
& \Lambda^{\prime}=-\Lambda \text { définie par (7) }
\end{aligned}
$$

$\mathbf{F}(t)$, vecteur des "forces aléatoires", de valeur moyenne nulle, est tel que (B3) vérifie (B1) compte tenu de (B2).

D'autre part, la fonction de corrélation de $\mathbf{F}(t)$ a pour valeur :

$$
\left\langle\mathbf{F}(t) \cdot \mathbf{F}^{T}(u)\right\rangle=\langle\Sigma\rangle . \delta(t-u)
$$

avec $\delta(t-u)$ fonction delta de Dirac.

La transformée de Fourier des fonctions de corrélation vaut par définition :

$$
s_{k l}(f)=\int_{-\infty}^{\infty} \varphi_{k l}(\tau) \cdot \mathrm{e}^{-i 2 \pi f \tau} \cdot \mathrm{d} \tau
$$

avec :

$$
k, l=N, C, \theta_{\mathrm{u}} .
$$

A l'aide de (B3), (B5) et (B6), nous pouvons montrer que [6] :

$$
\begin{aligned}
S(f)= & {\left[i(2 \pi f) . I+\Lambda^{\prime}\right]^{-1} \times } \\
\times & <\Sigma>\cdot\left[-i(2 \pi f) . I+\Lambda^{\prime}{ }^{T}\right]^{-1}
\end{aligned}
$$

avec :

I

$S(f)=\left[s_{k l}(f)\right]$ matrice des densités spectrales qui permet de calculer celles définies au chapitre 3.

\section{Bibliographie}

[1] Dalfes A., The Fokker-Planck and Langevin equations of a nuclear reactor; Nukleonik 5 (1963).

[2] Dalfes A., The correlation function and power spectral density of nuclear reactor; Nukleonik 6 (1964).

[3] LAX M., Classical noise IV : Langevin methods; Rev. Mod. Physics 38 (1966).

[4] Harris D. R., Stochastic fluctuations in a power reactor; WAPD-TM-190, Bettis Atomic Power Laboratory, 1958.

[5] Pluta P. R., Probabilistic analysis of reactor kinetics; AEC Symposium series $n^{\circ} 2,1964$.
[6] SAITo K., Input-noise source of at-power reactors with temperature feedback effect ; NSE, 37 (1969).

[7] SaIto K., Temperature noise in low-power reactor; NSE, 37 (1969).

[8] LAX M., Fluctuations from non-equilibrium steady state; Rev. Mod. Physics 32 (1960).

[9] LAX M., Classical noise III : Nonlinear Markoff Processus; Rev. Mod. Physics 38 (1966).

[10] Randall R. L. and Griffith C. W., Application of power spectra to reactor system analysis; AEC, $n^{\circ} 4,1964$. 